\title{
APOPTOTIC MACHINERY: THE BCL-2 FAMILY PROTEINS IN THE ROLE OF INSPECTORS AND SUPERINTENDENTS
}

\begin{abstract}
Aleš Tichý
Charles University in Prague, Faculty of Medicine in Hradec Králové, Czech Republic: Department of Medical Biochemistry

Summary: Programmed cell death, apoptosis, plays an integral role in a variety of biological events, e.g. morphogenesis, removal of unwanted or harmful cells, tissue homeostasis etc. Members of the Bcl-2 family have been described as the key players in the regulation of the apoptotic process. This family consists of proteins that prevent apoptosis (Bcl-2-like) and two structurally distinct subgroups (Bax-like and $\mathrm{BH} 3$-only) that on the contrary promote cell death. Majority of their response is concentrated to the mitochondrial level. In this paper, besides reviewing some new information in this field we focused on how they interact among each other and on the way they sense and influence the death signals from the environment. Here, we compare Bcl-2 family to inspectors and superintendents since they supervise the manufacturing process of cell death and they determine whether the cell will die or it will resist and survive.
\end{abstract}

Key words: Apoptosis; Bcl-2 family proteins; Mitochondria; Caspases

\section{Introduction}

Programmed cell death or apoptosis is a process of selfdestruction with distinctive morphological features, which is important to embryonic development, maintenance of homeostasis, and pathogenesis of many diseases $(48,50)$. Apoptosis is like a cellular death producing manufactory with a number of employees. Many workers such as caspase enzymes execute the job of the killers but it is up to the officers, who control the production and the managers who integrate the communication within the factory to decide whether the cell machinery will stop or continue working. One of these managers and officers are proteins of $\mathrm{Bcl}-2$ family.

To understand properly the particular functions of $\mathrm{Bcl}-2$ proteins we shall primarily describe from where the death signal comes from, how is it mediated and executed and then how Bcl-2 proteins affect it.

\section{Triggering the programmed death}

A great number of death stimuli might trigger a programmed cell death. They can induce a death signal, which further expands by at least two caspase-dependent signalling pathways. The death receptor pathway is usually discussed as the first one. Members of TNF (Tumour Necrosis Factor) super family trigger apoptosis extracellularly. The second pathway is called mitochondrial.

\subsection{Death receptor pathway}

TNF-like members such as TNF $\alpha$, TRAIL (TNFRelated Apoptosis Inducing Ligand), FasL (also called CD95L) and TWEAK (TNF-like weak inducer of apoptosis) also designated as death ligands (25) bind to specific death receptors (DR) integrated in cytoplasmic membrane, thus inducing DR trimerisation. This causes a recruitment of adaptor proteins TRADD (TNF-Receptor-1 Associated Death Domain) and/or FADD (Fas-Associated Death Domain), which happens via 70-80 amino acid interaction motifs, called the death domain. TRADD and FADD contain another motif, called the death effector domain (DED) which recruits an immature protease, procaspase- 8 (see below), leading to the formation of the death inducing signalling complex (DISC).

\subsubsection{Caspase family}

An essential step in the execution phase of the apoptotic death program involves the sequential cleavage and activation of a hierarchical cascade of cystein-aspartate proteases, caspases, which are the key workers of the apoptotic machinery.

They are minimally activated in the healthy cells being synthesized as zymogens, which need further enzymatic activation at specific aspartate residues to fulfil their function. Caspases can be classified as the initiators of apoptosis, including so-called apical or upstream caspase-2, -8, -9, -10 and, probably, -11 and the executioners of apoptosis 
(downstream caspases), which are caspase-3, -6 and -7 (35). The initiator caspases appear at the beginning of the death signal pathway and they are characterised by presence of an extended $\mathrm{N}$-terminal caspase recruitment domain (CARD) or DED (see above). In order to be activated caspases bind by these domains to the specific scaffold or adaptor proteins (e.g. TRADD, FADD) whose task is to ensure close proximity permitting proteolytical auto processing of molecules of procaspases (39).

Effector caspases or executioners of apoptosis, in turn, do that crucial job and cleave the vital cellular substrates and they are responsible for the nucleus breakdown and characteristic morphological and biochemical features of apoptosis (formation of apoptotic bodies, exposure of phophatidylserine at the surface of the cytoplasmic membrane as a signal to macrophages for subsequent phagocytosis etc.).
By the proteolytic cleavage in DISC procaspase- 8 becomes the mature initiator caspase- 8 that transmits and amplifies the death-inducing signal to other downstream executioner caspases such as caspase-3, -6 and -7 .

In contrast to mitochondrial pathway, the death receptor pathway is out of control of Bcl-2 family proteins, although the simple model of TNF-like members-mediated apoptosis is complicated by the existence of an amplification loop in cases of low initial caspase- 8 activation. In this scenario, caspase- 8 activates the pro-apoptotic $\mathrm{BH} 3$-only protein Bid that translocates to mitochondria and induces cytochrome c release (3); see Activation of $\mathrm{BH} 3$-only death factors.

Receptor pathway can be inhibited by the caspase- 8 homologue FLIP which contains its own DED and it can interact with adaptor proteins but it can not transmit the death signal because it is not capable of cleavage of other caspases (29). Alternatively, a competitive inhibition might

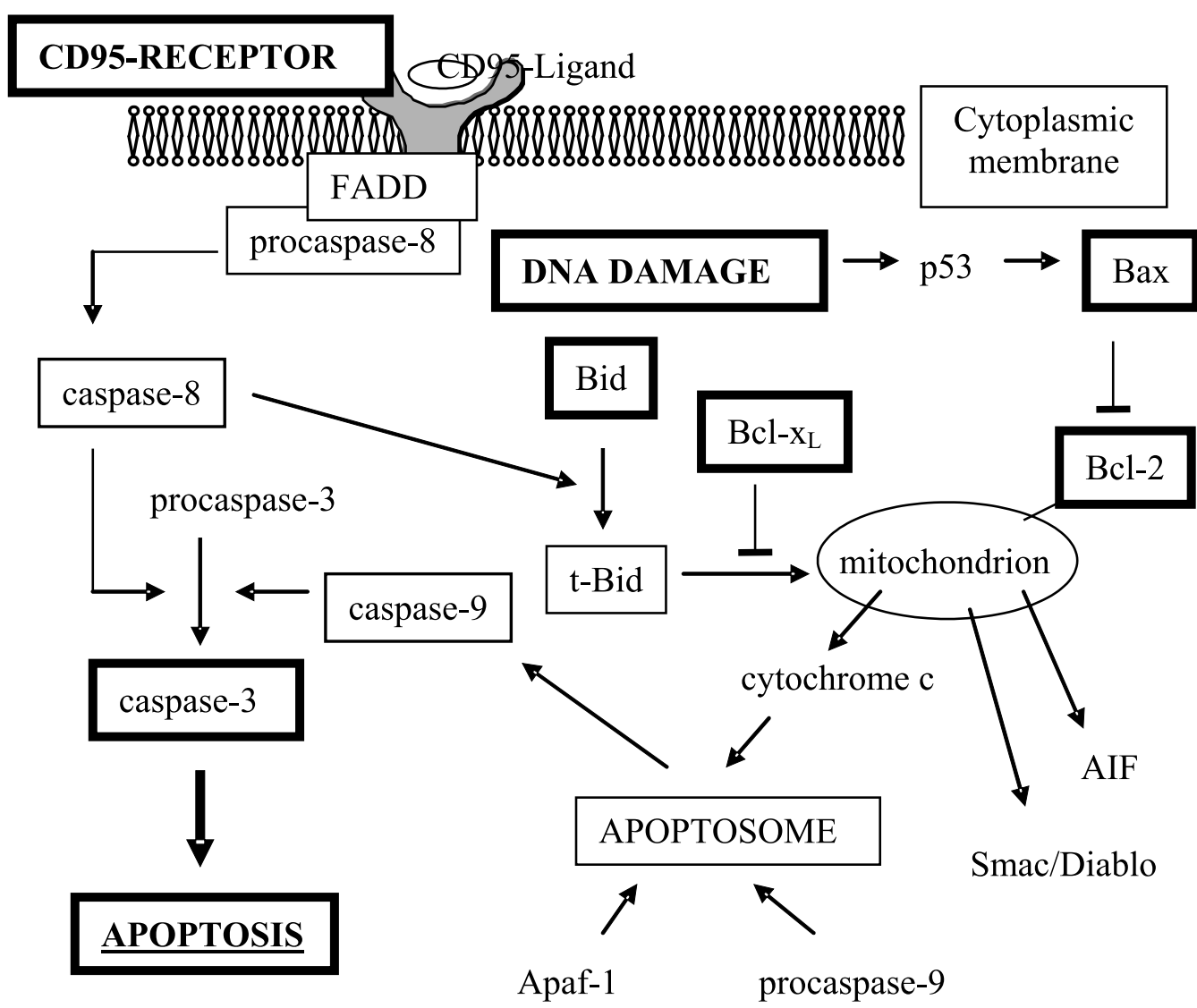

Fig. 1: Schematic model of apoptotic signalling pathways leading to activation of caspase-3. In the death receptor pathway plays the key role caspase- 8 , which is activated in the death inducing signalling complex localized near the cytoplasmatic membrane. Mature caspase- 8 then cleaves either procaspase- 3 or (in the case of its low activation) Bid (22 kDa) into t-Bid $(15 \mathrm{kDa})$. $\mathrm{t}$-Bid translocates to mitochondria where it induces changes of mitochondrial membrane with subsequent release of cytochrome $\mathrm{c}$. The mitochondrial pathway is under control of Bcl-2 proteins. The activation of Bax-like proteins leads as well to mitochondrial membrane permeabilization. Bcl-2-like survival factors block these events by binding Baxlike and BH3-only proteins either from mitochondria or ER. The release of cytochrome c later results in activation of caspase-9, which together with caspase- 8 activates the main executioner of apoptosis, caspase-3. For more details see text (according to 18). 
appear as an action of inhibitor of apoptosis proteins (IAPs), which compete with caspases at the active sites of their substrates (43).

\subsection{Mitochondrial pathway}

Several death receptor-independent apoptotic stimuli can contribute to triggering mitochondrial pathway: UVand $\gamma$-irradiation, chemotherapeutic drugs, viruses, bacteria, withdrawal of cytokines, neutrophins and growth factors or anoikis - detachment from the extracellular matrix (5). As Scaffidi et al. (42) reported, under certain circumstances (especially in some so-called type II cells) TNF-like factors can also trigger mitochondrial pathway in order to amplify the death signal in the case that caspase- 8 is minimally activated (see Activation of BH3-only death factors).

Each of these stimuli targets certain cellular components and transmits the death signal up to mitochondria. We still know little about the exact mechanism, but mitochondria must play a central role in this pathway, since it is sure that the outer membrane gets permeable for some proteins of the mitochondrial intermembrane space, which further plays a crucial role in activation of the main downstream caspases. It is cytochrome $\mathrm{c}$, which is probably the most important protein involved. When cytochrome $\mathrm{c}$ is released from mitochondria it binds WD-40 repeats of another protein, Apaf-1, thus triggering its ATP-dependent oligomerization and formation of so-called apoptosome, a large apoptosomal complex of 1.4 MDa. This complex recruits procaspase- 9 and allosterically enhances (by about 1000-fold) its activity, which leads to effective cleavage of caspase-3 and caspase-7 (40).

Bcl-2 family members do not control only the release of cytochrome $\mathrm{c}$ from the outer mitochondrial membrane, but also almost 30 other proteins (33). One of them is the "Second mitochondria-derived activator of caspase" (Smac) and serine protease $\mathrm{Htr} 2 \mathrm{~A} / \mathrm{Omi}$ whose task is to ensure apoptosome formation by triggering sequestration and/or degradation of cytosolic proteins - inhibitors of apoptosis (IAPs) - preventing their caspase inhibitory function (49). Anyway, the most important regulators of mitochondrial apoptotic pathway are Bcl-2 proteins. How do they influence mitochondria and what is the mode of their action?

\section{Bcl-2 protein family}

In higher eukaryotes this family involves up to 30 homologues that can have absolutely different impact on the cell fate by promoting or inhibiting apoptosis depending on their structural features. These features are Bcl-2 homology (BH) domains being highly conserved throughout this family. The pro-survival proteins contain $\mathrm{BH} 1, \mathrm{BH} 2, \mathrm{BH} 3$ and $\mathrm{BH} 4$ domain, whereas the pro-death members have, at least, a BH3 domain (17). The Bcl-2 family classification derives from homologues encoded in Caenorhabditis elegans, a nematode on which the first apoptotic studies were performed.
Bcl-2 proteins can be grouped in three categories: (i) Bcl-2-like survival factors such as Bcl-2, Bcl-X ${ }_{\mathrm{L}}$, Bcl-w, Mcl-1, A1/Bfl-1, NR-13, Boo/Diva/Bcl-2-L-10 and Bcl-B; (ii) proapoptotic BH3-only death factors such as Bik/Nbk, Blk, Hrk/DP5, BNIP3, Bim $/$ /Bod, Bad, Bid, Noxa, PUMA/ $\mathrm{Bbc} 3$ and Bmf; and (iii) pro-apoptotic Bax-like factors Bax, Bak, Bok/Mtd, Bcl-x $\mathrm{s}_{\mathrm{s}}$ and Drosophila DEBCL, a subgroup not presented in C. elegans (5).

\subsection{Bcl-2-like survival factors}

Every Bcl-2 like survival factor contains three to four homology domains (BH1-BH4). The BH1-BH3 domains form the hydrophobic pocket and the N-terminal $\mathrm{BH} 4$ domain stabilizes the protein structure from the backside (2). Since Petros et al. (34) referred about the NMR structure of Bcl- $\mathrm{x}_{\mathrm{L}}$ complex with the $\mathrm{BH} 3$ domain of the death factors Bak and $\mathrm{Bad}$, we know that $\mathrm{BH} 3$ domain is a random coil when free in solution, but it adopts an amphiphatic $\alpha$-helix when completed with another protein member. Therefore it just fits into the hydrophobic pocket, thus forming a heterodi/oligomer.

However, there are reports indicating that mode of action of Bcl-like survival factors is tricky and more complex. First, BH3 domains are not available for binding in all proteins at all times needing a post-translation modification and/or a conformational change (41; see Bax-like and BH3only death factors below). Second, a plenty of proteins (such as R-Ras, Raf-1, calcineurin, Bap31, BAG-1/Hsc70 or p53 binding protein - p53BP-2) without BH3 domain were shown to bind Bcl-2 survival factors (38). Third, solution of $\mathrm{Bcl}-2$ and $\mathrm{Bcl}-\mathrm{x}_{\mathrm{L}}$ structure revealed surprising structural homology with bacterial pore-forming toxins such as colchicin and diphteria toxin $(2,34)$, so it is likely that Bcl-2-like proteins use part of the hydrophobic pocket for other purposes than binding, namely for formation of ion- or protein-conducting channels (45). Finally, Bcl-2 was shown to prevent lipid peroxidation by scavenging oxygen radicals functioning as an antioxidant (19) or by inhibition of caspases involved in production of oxygen radicals. In addition, we also have to consider regulatory effects of proteins that are not presented under in vitro binding conditions.

Briefly, Bcl-2-like survival factors tail-anchored in intracellular membranes (mitochondrial, nuclear, endoplasmic reticulum) can scavenge pro-apoptotic death factors (and even some BH3-lacking proteins) and in contrast to the death factors they do so without any major change in conformation or subcellular localization (5).

\subsection{Bax-like death factors}

This subgroup consists of four members. The first one isolated was named Bax for Bcl-2-associated protein $\mathrm{X}$, since it immunoprecipitated together with Bcl-2 and blocked its survival activity when co-expressed (31). Two other homologues were isolated in mammals, Bak and Bok/Mtd (16, $20)$ and one in Drosophila, Drob/dBorg/DEBCL $(7,11)$. 
Bax-like death factors comprise $\mathrm{BH} 1-\mathrm{BH} 3$ domains and their pro-apoptotic behaviour was originally associated with the lack of stabilizing BH4 domain as its absence could unfold the hydrophobic pocket and trigger conformational changes conferring pro-apoptotic activity. Nevertheless, there are some cellular proteins such as Mcl-1 or A1 and all viral homologues lacking $\mathrm{BH} 4$ region and they are potent cell survival and not death factors (1). Moreover, Bcl- $\mathrm{x}_{\mathrm{L}}$ lacks $\mathrm{BH} 1$ and $\mathrm{BH} 2$ but retains $\mathrm{BH} 4$ and possesses pro-survival activity (4).

\subsubsection{Mitochondrial targeting}

The diversity of the BH3-region helix seems to be crucial for mitochondrial targeting and later pro-apoptotic activity. It is less packed to the hydrophobic pocket in Bax than in $\mathrm{Bcl}-\mathrm{x}_{\mathrm{L}}$ and therefore more available for binding to the hydrophobic grooves of Bcl-2-like survival factors (41). Another difference consists in the fact that hydrophobic membrane-anchoring C-terminus $(\alpha 9)$ of $\mathrm{Bcl}-2$ is exposed immediately after proteosynthesis and needs to be targeted to membranes instantly in order to prevent clustering and precipitation. On the other hand, $\alpha 9$ terminus of molecule of Bax is folded back in the hydrophobic groove and therefore it is protected from binding to membranes as well as to other proteins (30) at least until $\alpha 9$ is exposed.

Mitochondrial targeting of Bax is determined by some yet unknown protein or post-translational modification that uncovers $\alpha 9$, but we still do not fully understand the mechanism of membrane targeting, insertion, oligomerization and channel formation on molecular level. One mode proposes that Bax is weakly attached to mitochondrial membrane, with intact hydrophobic pocket and it is kept inactive by an unknown inhibitory protein or by a specific lipid. Another model involves conformational changes and exposure of $\mathrm{BH} 3$ domain which enables either Bcl-2-like factors inhibition of Bax and cell survival upon death signal or stable membrane insertion of Bax, oligomerization and/or interaction with VDAC/ANT (voltage-dependent anion channel, adenosine nucleotide transporter). Subsequent pore or channel formation releases cytochrome c and triggers caspase cascade (5).

\subsubsection{Mode of action of Bax-like death factors is not fully} elucidated

Mitochondria have been the focus of the majority of studies aimed at explaining the role of the Bcl-2 family in cell death. Evidences for such a role are: loss of mitochondrial potential and release of pro-apoptotic factors including cytochrome c, AIF and caspase activation upon disruption of mitochondrial membrane (17) which probably involves channel formation and its opening rather than membrane rupture. It is not clear whether Bax directly forms a channel or interacts with a pre-existing one. Even number of investigators $(32,28,44)$ has not found an importance of PT pore for the cytochrome c-releasing activity of Bax. On the other hand, such a channel might be permeability transi- tion (PT) pore whose main components are voltage-dependent anion channel (VDAC) in the outer membrane, adenosine nucleotide transporter (ANT) in the inner membrane and cyclophilin $\mathrm{D}$ in the matrix. This channel allows passage of molecules up to $1.5 \mathrm{kDa}$, therefore one hypothesis is that Bax would interact with PT pore increasing its size. Then even higher molecular weight molecules (up to $15 \mathrm{kDa}$ ) as cytochrome c can pass through (54).

In summary, Bax-like death factors either form channels or interact with channel-forming proteins, and as Korsmeyer (21) proposed, Bcl-2 survival factors (tail-anchored to different intracellular membranes) sequester Bax-like factors, thus co-working like a survival/death rheostat. Nevertheless, the exact mode of the Bax-like death factors action stays controversial.

\subsection{BH3-only death factors}

The BH3-only death factors share only the short $\mathrm{BH} 3$ domain with each other and with the rest of the Bcl-2 family. They function as mediators of death signalling and sensors for cellular integrity (e.g. Bim for cytoskeleton integrity, Bad for growth factor withdrawal and Bid as a sensor for death receptor pathway signalling; see below). BH3-onlies are kept in an inert state preventing inappropriate cell death and one or several of the following mechanisms activate them.

\subsubsection{Activation of BH3-only death factors}

Each of mammalian $\mathrm{BH} 3$-onlies is regulated differently, depending on the nature of the protein and the origin of apoptotic stimulus. One mechanism is transcriptional induction. For example p53 is a transcription factor that induces PUMA/Bbc 3 and Noxa after DNA damage affected by chemotherapeutics, UV- and $\gamma$-irradiation (22).

Cytokine or growth factor withdrawal, anoikis and death receptor ligation induce a second possible way of BH3onlies activation - post-translational modification phospho- rylation/dephosphorylation. For instance, phosphorylated Bad is inactive and sequestered in cytoplasm by binding to 14-3-3 scaffold protein (55). Dephosphorylation of Bad processed by calcineurin (51) makes Bad free and available for interaction with Bcl-2-like survival factors, thus triggering apoptosis.

Another mechanism is based on proteolysis; it involves Bid when the death receptor pathway is not fully activated. Active caspase- 8 cleaves Bid ( $22 \mathrm{kDa}$ ) into a truncated fragment, tBid ( $15 \mathrm{kDa})$, thereby exposing a binding site for $\mathrm{N}$-myristoylation (56), which together with its high affinity to mitochondrial cardiolipin (26) causes targeting of tBid to mitochondria (24). tBid seems to change lipid composition of the outer mitochondrial membrane (15), making it more permeable during apoptosis. Besides that, tBid is able to release Bax-like factors from Bcl-2-like factors as well as to stimulate oligomerization and membrane insertion of Bax and Bak (14,52). Pro-apoptotic role of Bid is undeniable, but probably cell-type dependent (53). Moreover, Bid 
can be cleaved also by other caspases (24) and even by noncaspase proteases e.g. cathepsin B (46), but so far this often occurs after mitochondria perforation and probably serves as a positive feedback loop.

Lastly, BH3-onlies are bound in the inactive state to the important macromolecular structures such as microtubules being placed as the stress sensors that are released after apoptotic stimuli. For instance, Bim is bound to a light chain of dynein motor complex on microtubules. Taxol, a microtubule-polymerising drug, can trigger the release of $\mathrm{Bim}$ and its association with Bcl-2/ $\mathrm{Bcl}_{\mathrm{L}}(36)$.

In summary, once $\mathrm{BH} 3$-onlies are post/translationally activated, they interplay with Bcl-2-like survival factors. They inactivate Bcl-2-like survival factors that restrict Baxlike death factors oligomerization. These free pro-apoptotic factors undergo conformational changes, subsequently disturb the outer mitochondrial membrane and release caspase-activating and other pro-apoptotic factors (23).

\section{Conclusion: Bcl-2 inspectors and superintendents}

In the cell death producing machinery Bcl-2 family proteins are working on the very important posts. The fact that they exist as inactive conformers needing activation by death signals suggests that members of this family are strategically positioned within the cell (just like the inspectors) to record damage at the specific sites and to rapidly converge diverse cell injuries on the common apoptotic pathway (8). In mammals, the execution of the most of so-called "death orders" takes place by the central part of the manufactory - the mitochondrion. This is the target of the Bcl-2 family proteins, the mediators of apoptosis. Their major function is to directly control the outer mitochondrial membrane permeability and subsequent release of several pro-apoptogenic factors (cytochrome c, Smac etc.) although the exact mechanism is still to be determined.

Subcellular distribution of Bcl-2 family proteins no doubt plays a crucial role in their ability to regulate apoptosis. Some of these superintendents ( $\mathrm{Bcl}-2)$ are also localized on endoplasmic reticulum serving as a first line of defence against apoptotic signals (5). They sequestrate proapoptotic proteins keeping them away from their site of action (the mitochondria). On the other hand, Bcl-2 and Bcl- $\mathrm{x}_{\mathrm{L}}$ on the mitochondria inhibit the death factors that have already accumulated there (47) functioning as a second defence line.

\section{Outlook}

Recent genetic and biochemical studies $(6,10,37)$ showed that $\mathrm{Bcl}-2$ proteins act in coordinated and interdependent manner, since Bax- and Bak-like pro-apoptotic proteins are not able to execute death in the cells where $\mathrm{BH} 3$-only proteins have not been activated in response to the stress stimuli. Despite of increasing number of publications and new findings about the interactions among Bcl-2 family proteins we are still far from full understanding the complexity of the controlling pathways. Basically, BH3-only proteins (activated via one of the mechanisms mentioned above) can act through Bcl-2 and Bax subfamilies because they contain the binding site for $\mathrm{BH} 3$-only proteins (the hydrophobic pocket). However, this fact contradicts a variety of recent findings, which might be misguiding. Most of the contemporary studies have been done with over expressed proteins and the binding affinities among particular Bcl-2 proteins have not been determined yet. Therefore, we can hardly say which interactions might occur under the physiological conditions. Hence, only some general models of the mode of the Bcl-2 family action can be proposed.

We are still dealing with the questions like what mechanisms induce the loss of mitochondrial integrity, how the intramitochondrial proteins are released, what role plays calcium in this process etc. Answering such questions will surely help us to find new therapeutic attitudes $(9,12,27)$ and it would be probably of great implications because physiological apoptosis is essential for healthy development and homeostasis of mammals.

Dysregulation of programmed cell death leads to various diseases in humans, including cancer, certain neurodegenerative diseases etc. For example, failure to remove autoimmune cells (that arise during development or that develop as a result of somatic mutation during an immune response) can result in autoimmune diseases or (in the case of damaged or mutant cells) in cancer. Therefore compounds that block survival and activate pro-apoptotic proteins as recently discovered $\mathrm{BH} 3$-mimetics (13) could offer a new way to treat cancer and other diseases. This way would be targeted at $\mathrm{Bcl}-2$ proteins family but a lot of further work in this field is needed.

\section{Acknowledgement}

Thanks are due to Professor Jirina Vavrova and Assoc. Professor Jaroslav Cerman for helpful discussion and critically reading this review and their encouragement to write this paper. Supported by project MSM 0021620820, Ministry of Education, Czech Republic.

\section{References}

1. Adams JA, Corry S. The Bcl-2 protein family: arbiters of cell survival. Science 1998;281:1322-6.

2. Aritomi M, Kunishima N, Inohara N, Ishbashi $\mathrm{Y}$, Ohta $\mathrm{S}$ and Morikawa $\mathrm{K}$ Crystal structure of rat $\mathrm{Bcl}-\mathrm{x}_{\mathrm{L}}$ : implications for the functions of the $\mathrm{Bcl}-2$ protein family. J Biol Chem 1997;272:27886-92.

3. Belka C, Budach W. Anti-apoptotic Bcl-2 proteins: structure, function and relevance for radiation biology. Int J Radiat Biol 2002;78(8):643-58.

4. Boise LH, Gonzales-Garcia M, Postema CE et al. Bcl-x, Bcl-2 related gene that functions as a dominant regulator apoptotic cell death. Cell 1993;74:597-608.

5. Borner $\mathrm{C}$. The Bcl-2 protein family: sensors and checkpoints for life-or-death decisions. Mol Immun 2003;39:615-47.

6. Bouillet P, Strasser A. BH3-only proteins - evolutionary conserved pro-apoptotic Bcl-2 family members essential for initiating programmed cell death. J Cell Science 2002;115:1567-74.

7. Brachmann CB, Jassim OW, Waschmuth BD, Cagan RL. The drosophila Bcl-2 family member $\mathrm{dBorg} 1$ functions in the apoptotic response to UV-irradiation. Curr Biol 2000;10:547-50. 
8. Burlacu A. Regulation of apoptosis by Bcl-2 family proteins. J Cell Mol Med 2003;7(3):249-57.

9. Cerman J, Ćáp J, Mareková M et al. The role of apoptosis in pituitary adenomas in the field of conventionally used therapeutic approaches. Annals NY Acad Sci 2003;1010:520-4.

10. Cheng $\mathrm{EH}$, Wei MC, Weiler $\mathrm{S}$ et al. Bcl-2 and Bcl- $\mathrm{x}_{\mathrm{L}}$ sequester $\mathrm{BH} 3$ domain-only molecules preventing Bax- and Bak-mediated mitochondrial apoptosis. Mol Cell 2001;8:705-11.

11. Colussi PA, Quinn LM, Huang DC et al. Debcl, proapoptotic Bcl-2 homologue is a component of the Drosophila melanogaster cell death machinery. J Cell Bio 2000;148:703-14.

12. Črvinka M, Cerman J, Rudolf E. Apoptosis in Hep-2 cells treated with etoposide and colchicine. Cancer Detect Prev 2004;28:214-26.

13. Degterev A, Lugovskoy A, Cardone $M$ et al. Identification of small-molecule inhibitors of interaction between the BH3 domain and Bcl-xL. Nat Cell Biol 2001;3(2): 173-82.

14. Desagher S, Osen-Sand A, Nichols A, Eskes R, Monetssuit S, Lauper S. Phosphorylation of Bid by casein kinase I and II regulates its cleavage by caspase-8. Moll Cell 1999;8:601-11.

15. Esposti MD. Sequence and functional similarities between pro-apoptotic Bid and plant lipid transfer protein. Biochim Biophys Acta 2002;1553:331-40.

16. Farrow SN, Brown R. New members of the Bcl-2 family and their protein partners. Curr Opin Genet Dev 1996;6:45-9.

17. Fridman JS, Parsels J, Rehemtulla A, Maybaum J. Cytochrome c depletion upon expression of Bcl-XS. J Biol Chem 2001;276:4205-10.

18. Hengartner MO. The biochemistry of apoptosis. Cell 1993;75:241-51.

19. Hockenbery DM, Oltvai ZN, Yin XM, Miliman C, Korsmeyer SJ. Bcl-2 function in an antioxidant pathway to prevent apoptosis. Cell 1993;75:241-51.

20. Inohara N, Ekhterae D, Garcia I, Carrio R, Merry A, Chen S. Mtd, a novel Bcl-2 family member activates apoptosis in the absence of heterodimerization with $\mathrm{Bcl}$ 2 and Bcl- $\mathrm{x}_{\mathrm{L}}$. J Biol Chem 1998;273:8705-10.

21. Korsmeyer SJ. Bcl-2 initiates a new category of oncogenes: regulators of cell death Blood 1992;80:879-86.

22. Lakin ND, Jacskon SP. Regulation of p53 in response to DNA damage. Oncogene 1999;18:7644-55.

23. Letai A, Bassik M, Walensky L, Sorcinelli M, Weiler S, Korsmeyer S. Distinct $\mathrm{BH} 3$ domains either sensitize or activate mitochondrial apoptosis, serving as prototype cancer therapeutics. Cancer Cell 2002;2:183-92.

24. Li H, Zhu H, Xu CJ, Yuan J. Cleavage of Bid by caspase-8 mediates the mitochondrial damage in the Fas pathway to apoptosis. Cell 1998;94:491-501.

25. Locksley RM, Killeen M, Lenardo MJ. The TNF and TNF superfamilies: integrating mammalian biology. Cell 2001;104:487-501.

26. Lutter M, Fang M, Luo X, Nishijima M, Xie X, Wang X. Cardiolipin provides specifity for targeting of tBid to mitochondria. Nat Cell Biol 2000;2:754-61.

27. Mareková M, Čáp J, Vokurková D, Cerman J. Effect of therapeutic doses of ionising radiation on the somatomammotroph pituitary cell line, GH3. Endocrine J 2003;50:621-8.

28. Marzo I, Brenner C, Zamzami $\mathrm{N}$ et al. The permeability transition pore complex: a target for apoptosis regulation by caspases and bcl-2-related proteins. J Exp Med 1998;187(8):1261-71.

29. Medema JP, Scaffidi C, Kischkel FC et al. FLICE is activated by association with the CD95 death-inducing signalling complex (DISC). EMBO J 1997;16: 2794-804.

30. Nechushtan A, Smith CL, Hsu Y-T, Youle RJ. Confirmation of the Bax C-terminus regulates subcellular location and cell death. EMBO J 1999;18:2330-41.

31. Oltvai ZN, Milliman CL, Korsmeyer SJ. Bcl-2 heterodimerizes in vivo with a conserved homolog, Bax, that accelerates programmed cell death. Cell 1993;74 609-19.

32. Pastorino JG, Tafani M, Rothman RJ, Marcineviciute A, Hoek JB, Farber JL. Functional consequences of the sustained or transient activation by Bax of the mitochondrial permeability transition pore. J Biol Chem 1999:274(44):31734-9.

33. Patterson SD, Spahr CS, Daugas E et al. Mass spectrophotometric identification of proteins released from mitochondria undergoing permeability transition. Cell Death Differ 2000;7:137-44

34. Petros AM, Medek A, Nettesheim DG et al. Solution structure of the anti-apoptotic protein bcl-2. Proc Natl Acad Sci USA 2000;98:3012-7.

35. Philchenkov A. Caspases: potential targets for regulating cell death J Cell Mol Med 2004;8(4):432-44
36. Puthalakath H, Huang DCS, O'Reilly LA, King SM, Strasser A. The pro-apoptotic activity of the Bcl-2 family member Bim is regulated by interaction with dynein motor complex. Mol Cell 1999;3:287-96.

37. Rathmell JC, Thompson CB. Pathways of apoptosis in lymphocyte development homeostasis, and disease. Cell 2002;109(suppl):S97-107.

38. Reed J. Bcl-2 family proteins. Oncogene 1998:17:3225-36.

39. Robertson JD, Enoksson M, Suomella M, Zhivotovsky B, Orrenius S. Caspase-2 acts upstream of mitochondria to promote cytochrome $\mathrm{c}$ release during etoposide-induced apoptosis. J Biol Chem 2002;277:29803-9.

40. Rodriguez J, Lazebnik Y. Caspase-9 and Apaf-1 form an active holoenzyme. Genes Dev 1999;13:3179-84

41. Sattler M, Liang H, Nettesheim D et al. Structure of Bcl-x-Bak peptide complex: recognition between regulators and apoptosis. Science 1997;275:983-6.

42. Scaffidi C, Fulda S, Srinivasan A et al. Two CD95 (APO-1/Fas) signaling pathways. EMBO J 1998;17:1675-87.

43. Shi Z. Mechanisms of caspase activation and inhibition during apoptosis. Mol Cell 2002:9:459-70

44. Shimizu S, Tsujimoto Y. Proapoptotic BH3-only Bcl-2 family members induce cytochrome c release, but not mitochondrial membrane potential loss, and do not directly modulate voltage-dependent anion channel activity. Proc Natl Acad Sci USA 2000;97(2):577-82.

45. Schendel SL, Azimov R, Pawlowski K, Godzik A, Kagan BL, Reed JC. Ion channel activity of the BH3-only Bcl-2 family member, Bid. J Biol Chem 1999; 274:21932-6.

46. Stoka V, Turk B, Schendel SL et al. Lysosomal proteass pathways to apoptosis. Cleavage of Bid, not pro-caspases is the most likely route. J Biol Chem 2001;276 3149-57.

47. Thomenius MJ, Clark WD. Bcl-2 on the endoplasmatic reticulum: protecting the mitochondria from a distance. J Cell Science 2003;116:4493-9.

48. Thompson CB. Apoptosis in pathogenesis and treatment of disease. Science 1995;267:1456-62.

49. Van Loo G, van Gurp M, Depuydt B et al. The serine protease Omi/HtrA2 is released from mitochondria during apoptosis. Omi interacts with caspase inhibitor XIAP and induces enhanced caspase activity. Cell Death Differ 2002; $9 \cdot 20-6$

50. Vaux DL, Korsmeyer SJ. Cell death in development. Cell 1999;96:245-54

51. Wang HG, Pathan N, Ethell IM et al. $\mathrm{Ca}^{2+}$ induced apoptosis through calcineurin dephosphorylation of Bad. Science 1999;284:339-43.

52. Wei MC, Lindsten T, Mootha VK et al. TBid, a membrane-targeted death ligand, oligomerizes Bak to release cytochrome c. Genes Dev 2000;14:2060-71.

53. Yin XM, Wang K, Gross A et al. Bid-deficient mice are resistant to Fas-induced hepatocellular apoptosis. 1999;400(6747):886-91.

54. Zamzami N, Kroemer G. The mitochondrion in apoptosis: how Pandora's box opens. Nat Rev Mol Cell Biol 2001;2:67-71.

55. Zha J, Harada H, Yang E, Jockel J, Korsmeyer SJ. Serine phosphorylation of death agonist Bad in response to survival factor results in binding to 14-3-3 not Bcl-x $x_{L}$. Cell 1996;87:619-28.

56. Zha J, Weiler S, Oh KJ, Wei MC, Korsmeyer SJ. Post-translational N-myristoylation of Bid as a molecular switch for targeting mitochondria and apoptosis. Science 2000;290:1761-5

Submitted July 2005

Accepted March 2006.

Mgr. Aleš Tichý,

Charles University in Prague,

Faculty of Medicine in Hradec Králové,

Department of Medical Biochemistry,

Simkova 870,

50038 Hradec Králové,

Czech Republic.

e-mail: tichya@lfhk.cuni.cz 